\title{
Commentary: Cardiovascular Comorbidities in a United States Patient Population with Hemophilia A: A Comprehensive Chart Review
}

Thomas J. Humphries*

44 Pinewood Drive, Neptune, NJ 07753, USA

Article Info

\section{Article Notes}

Received: September 22, 2018

Accepted: November 22, 2018

\section{*Correspondence:}

Dr. Thomas J Humphries, MD, FACP, 44 Pinewood Drive,

Neptune, NJ 07753, USA:

Email: humphries_993@hotmail.com.

C 2018 Humphries TJ. This article is distributed under the terms of the Creative Commons Attribution 4.0 International License.
A recent publication of a chart review of cardiovascular comorbidities in US persons with hemophilia A raises several questions ${ }^{1}$. These include why it was conducted, how do the results impact the hemophilia community, why the review did not confirm the findings of 2 prior very large, controlled commercial database reviews ${ }^{2,3}$, and what might be appropriate as next steps?

A prior study using The MarketScan $®$ Commercial and Medicare Research databases compared cardiovascular comorbidities in a large hemophilia A cohort $(n=2,506)$ to those in a 3:1 control cohort $(\mathrm{N}=7,518)$. The surprising result was that the prevalence's of eight comorbidities were significantly greater in the hemophilia A cohort as compared to controls. In most comorbidities, the significant differences were first seen in the youngest age brackets, 0-17 and 18-29 years of age as shown in Table 1 . These included ischemic and hemorrhagic stroke, and arterial and venous thrombosis ${ }^{2}$. Concern over these findings prompted a second large commercial database review using the PharMetrics $\AA$ LifeLink Claims database with an estimated overlap of $10 \%$. With exception of myocardial infarction and hyperlipidemia, the findings were strikingly similar ${ }^{3}$.

Because of known limitations of commercial databases which include the possibilities of coding errors including coding for ruleout rather than actual disease, and the fact that other potentially contributing cardiovascular conditions (diabetes, smoking, body

Table 1: Prevalence (\%) of four cardiovascular risk factors in hemophilia vs. controls over an age range of $1-49$ years $^{2}$

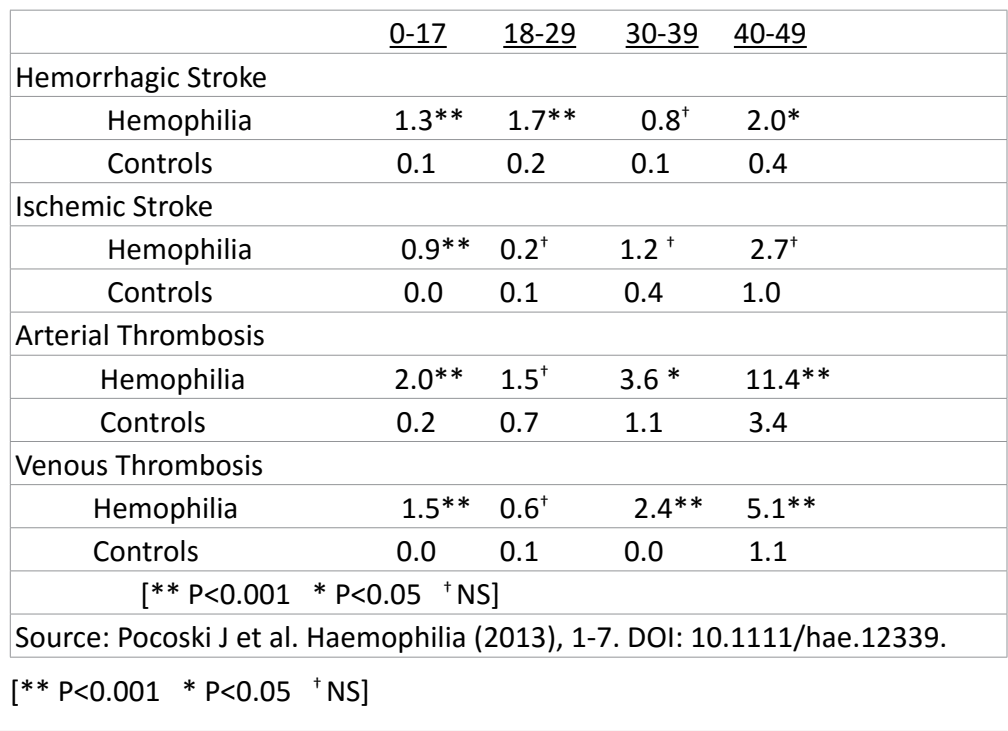


mass index) often are not counted, it was elected to do a comprehensive chart review at a large US institution. A hemophilia group $(\mathrm{N}=74)$ was compared to a matched control group from the general patient population $(\mathrm{N}=222)$. The two groups were generally well balanced with respect to age and race. The demographics in this study showed a higher percentage of African Americans than in other published studies. The data did not change when this group was excluded. In both groups, the patients were males. With respect to hemophilia-specific characteristics, hemophilia A severity was listed as severe in 52.7\%, $10.8 \%$ each for moderate and mild severity. Factor VIII treatment was documented for $83.8 \%$. With respect to clinical characteristics, as expected, type and number of bleeding events were noted almost exclusively in the hemophilia group. For non-cardiovascular comorbidities, the prevalence rates of hepatitis $\mathrm{B}$ and $\mathrm{C}$ and HIV/AIDS were significantly higher in the hemophilia group. HIV/ AIDS was the most prevalent comorbidity in both groups.

The prevalence rates of 12 cardiovascular comorbidities and associated risk factors were documented. For categorical variables, $\mathrm{P}$ values were generated from a chisquare test. For continuous variables, a $t$-test was used. In view of the small sample size, statistical differences were assessed using absolute standardized difference (SDiff). Measuring effect size with this approach is independent of sample size. Using these methods, the review generally did not confirm the findings of the 2 prior reviews ${ }^{2,3}$. The prevalence rates for hypertension, diabetes, obesity, hyperlipidemia, coronary artery disease, heart failure, stroke, venous and arterial thrombosis, ventricular arrhythmias, atrial fibrillation, and chronic renal disease were all numerically higher in the control group, but only diabetes $(\mathrm{P}=0.01)$ and hyperlipidemia $(\mathrm{P}=0.0001)$ were significantly greater. Meaningful statistical differences using standardized differences (SDiffs) were not reached for venous and arterial thrombosis and atrial fibrillation. A concern raised by the findings in the two prior reviews, the early age of appearance of comorbidities, was also seen in the chart review. In the hemophilia A group, hypertension and venous thrombosis first appeared in the 19-29-year age groups. In the controls, all comorbidities except arterial thrombosis also first surfaced in the 18-19-year age group! Possible reasons for the findings include different inclusion criteria from the prior studies, the facts that the control groups may have had differing medical burdens and the follow-up was for only one year.

In their paper reporting unfavorable cardiovascular disease risk profiles in a large cohort of Dutch and British hemophilia patients $(\mathrm{N}=709)$, van de Putte and colleagues cite the difficulties in discerning degree of risk for cardiovascular events in hemophilia ${ }^{4}$. Both decreases and increases in risk have been reported. Deficiencies in the literature included small numbers, often a lack of controls, and other methodological flaws that leaves the prevalence of cardiovascular risk factors unclear. The authors used the QRISK $^{\odot} 2$ in their cohort and found that the predicted ten-year risk was significantly higher in hemophilia patients than in the general population (8.9 vs. $6.7 \%, \mathrm{P}<0.001 \%$ ). Looking at individual risk factors, they found that hypertension was more common in hemophilia than controls, diabetes and smoking were similar, and obesity and hypercholesterolemia were lower. The age for entry was $\geq 30$ years, so this study did not address the risks in earlier age groups. Two additional papers also did not include patients under 35 , and also could not confirm the pattern seen in the chart review, the subject of this commentary ${ }^{5,6}$.

The findings in this set of 3 studies deserve confirmation, especially the aspect of early appearance of serious cardiovascular risks in both hemophilia A and controls. This has a potential impact on screening practices if confirmed. Addressing one cardiovascular comorbidity, an Editorial in a recent issue of JAMA has highlighted the data from several sources showing that high blood pressure in young adults increases the risk for premature cardiovascular disease ${ }^{7}$. This supports further evaluation of cardiovascular comorbidities in hemophilia. A possible approach would be to conduct a 3-5-year prospective, controlled evaluation of a large cohort of hemophilia A patients with attention to cardiovascular comorbidities by age groups. Careful selection of an adequate control group is a key factor, as is the quantification of the bleeding phenotypes in the hemophilia group. Such a study might be best conducted by organization seeing large numbers of hemophilia A patients, such as the National Hemophilia Foundation.

\section{References}

1. Humphries TJ, Rule B, Ogbonnaya A, et al. Cardiovascular comorbidities in a United States patient population with hemophilia A: A comprehensive chart review. Advances in Medical Sciences. 2018; 63: 329-333.

2. Pocoski J, Ma A, Kessler CM, et al. Cardiovascular comorbidities are increased in US patients with hemophilia A. Haemophilia. 2-14; 20 : 472-478.

3. Humphries TJ, Ma A, Kessler CM, et al. A second retrospective database analysis confirms prior findings of apparent increased cardiovascular comorbidities in hemophilia A in the United States. Amer J Hematol. 2016; 5: E297-299.

4. Fransen van de Putte DE, Fischer K, Mackris M, et al. Unfavourable cardiovascular disease risk profiles in a cohort of Dutch and British haemophlia patients. Thromb Haemost. 2013; 109:16-23.

5. Minuk L, Jackson S, Iorio A, et al. Cardiovascular disease (CVD) in Canadians with haemophila: Age-related CVD in Haemophilia Epidemiological Research (ARCHER study). Haemophilia. 2015; 1-6. DOI: $10.1111 /$ hae.12768.

6. Sharathkumar AA, Soucie JM, Trawinski B, et al. Prevalence and risk factors of cardiovascular disease (CVD) events among patients with 
haemophilia: experience of a single haemophilia treatment centre in the United States (US). Hemophilia. 2011; 1-8. DOI: 10.1111/j.13652516.2010.02463x
7. Vasan RS. High blood pressure in young adulthood and risk of premature cardiovascular disease. Calibrating treatment benefit to potential harm. JAMA. 2018; 320: 1760-1763. 